\title{
Shapes and motions of polysaccharide chains
}

\author{
David A. Brant \\ Department of Chemistry, University of California \\ Irvine, California 92697-2025 USA
}

\begin{abstract}
Computer-based molecular modeling of single stranded polysaccharide chains is capable of a quantitative description of the polymer chain dimensions in dilute aqueous solution. The influence of glycosidic linkage position and stereochemistry on the local chain trajectory is now well understood. Some illustrative results of modeling of this sort are reported here. More recent efforts to understand the possible influence of glycosidic linkage type on the dynamics of polysaccharide conformational change are also described.
\end{abstract}

\section{INTRODUCTION}

Important differences between polysaccharides and other biopolymers arise from the multiple hydroxyl functionality of the monomeric sugar units. Thus, aldohexopyranose sugars may be linked $(1,2),(1,3)$, and $(1,4)$ in a polysaccharide chain. The glycosidic linkage oxygen may have equatorial or axial disposition with respect to each sugar ring, depending on the anomeric form and chemical identity of the sugars involved. Together, the regio- and stereochemistry of the linkage govern the extent of steric conflict between successive sugar residues, which in turn determines the inherent conformational freedom of a given linkage. These same factors govern the general characteristics of the polysaccharide chain trajectory $(1)$, the equilibrium spatial distribution of the chain(2), the mean values of the characteristic metrics of that spatial configuration(3), and the rates of transformation beween conformations characteristic of that distribution(4). The possibility for extensive chain branching, which vastly expands the information carrying capacity of carbohydrate polymers and oligomers, is another important consequence of multiple hydroxyl functionality that will not be pursued here.

When aldohexopyranose sugars are linked $(1,6)$, interposition of an additional chemical bond in the linkage region between adjacent sugar residues promotes a greatly increased inherent conformational freedom as the two sugars are separated by a greater mean distance(5). Additional sources of chain tortuosity may also arise if there are several conformers of the pyranoid ring possessing similar energies, as in the L-iduronic residues of heparin(6). Sugar ring flexibility may play a much larger role in polysaccharides involving furanoid sugars owing to the well known propensity of furanoid sugars for pseudorotation(7). All of these factors conspire to make the polysaccharides more conformationally diverse than the other polymers of natural origin. Less well understood are the factors that determine the rates at which individual polysaccharide chains sample the conformational diversity to which they are entitled.

Whereas the proteins and nucleic acids possess substantial moieties that are hydrophobic in character, significant hydrophobic elements are less prevalent among the polysaccharides. These differences dictate that globular tertiary structures, generated by hydrophobic interactions of sequentially remote chain segments and characteristic of many proteins and some nucleic acids, are not observed among the polysaccharides. The widely recognized examples of polysaccharide helical chain folding and chain association are apparently mediated significantly by hydrogen bonded interactions of the sugar hydroxyls, even though the aqueous environment is not usually thought conducive to strong intramolecular hydrogen 
bonded interactions. While all proteins and nucleic acids are polyions, polysaccharides may or may not be ionic. The relatively high linear charge density of naturally occurring carbohydrate polyanions would also seem to militate against their association into tertiary and quaternary structural motifs. Nevertheless there are numerous well documented examples of multiple helical ionic polysaccharides that can be dispersed in aqueous solution with retention of the multiple helical order(8). Strong associative interactions must be at work to counter the electrostatic repulsions. This is another interesting aspect of polysaccharide conformation that cannot be explored here. Instead the focus will be on linear polysaccharide linkage position and stereochemistry, their relationship to the preferred spatial distribution of polysaccharide chains in aqueous solution, and the rates at which single stranded polysaccharide chains transform among the host of conformations that characterise this equilibrium spatial distribution.

\section{LOCAL STRUCTURE INFLUENCES LONG CHAIN CONFORMATION}

Insets in Figs. 1 and 2 show, respectively, dimeric segments of homopolymeric $(1,2)$ - and $(1,3)$-linked $\beta$-Dglucans. For present purposes it will suffice to treat the sugar rings as rigid and attribute all of the tortuosity of the dissolved glucans to the linkage torsion angles $\phi$ and $\psi$. The conformational freedom of a linkage is conveniently described with a contour map of the energy of the dimeric segment in $\phi, \psi$ space, as shown in Figs. 1 and 2. These maps are approximations based on commercial molecular mechanics software. Their features reflect primarily the interactions of the groups flanking the linkage. Regions of low energy are contoured; higher energy domains are left vacant to signify their inaccessability at normal thermal energies. The near identity of the maps in Figs. 1 and 2 is expected from the structural similarity of the dimeric units in the vicinity of the linkage. At room temperature each linkage is confined effectively to the region of $\phi, \psi$ space within $3 \mathrm{kcal} / \mathrm{mol}$ of the energy minimum located at the position marked $\mathrm{X}$. Thus, although these linkages cannot explore much of the conformational space potentially available, they are by no means confined to a single conformation. Any complete modeling of the behavior of these molecules must therefore invoke the methods of statistical mechanics as well as those of molecular mechanics $(3,9)$.
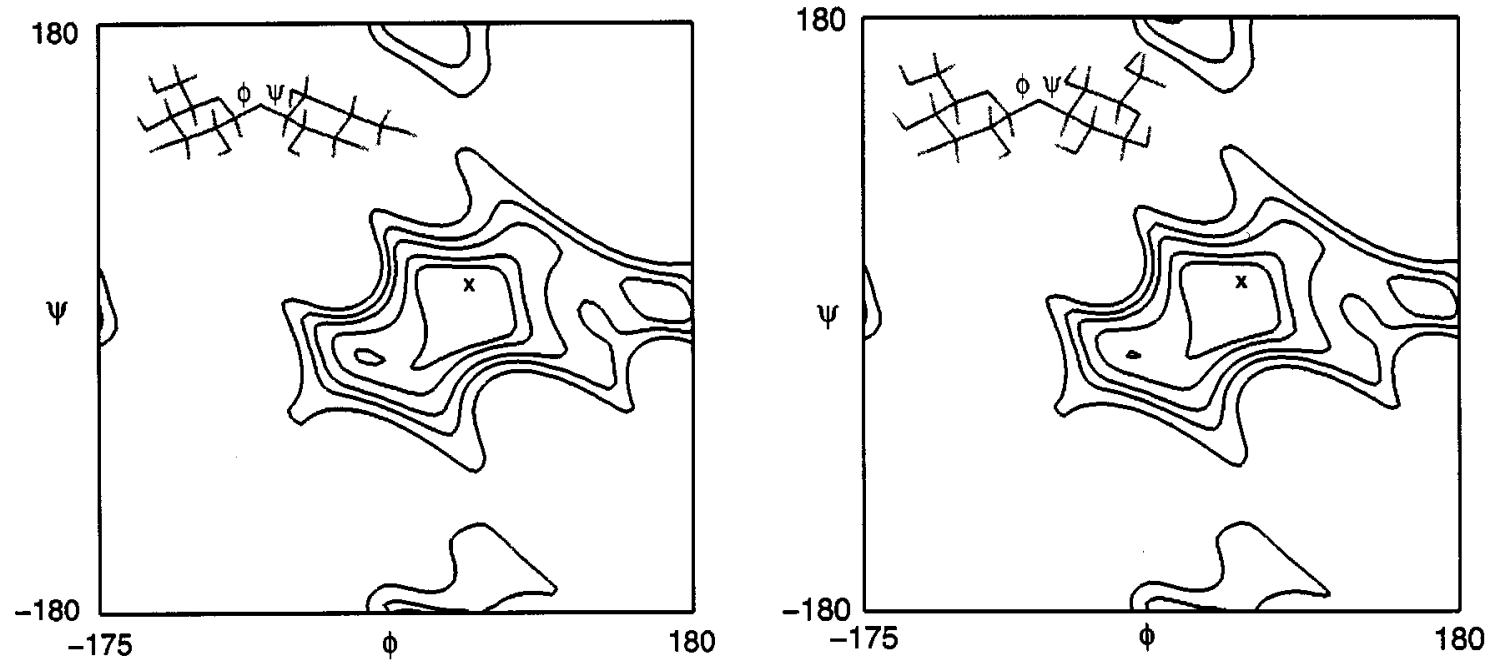

Fig. 1 (left). Conformational energy surface for dimeric segment of homopolymeric $(1,2)$-linked $\beta$-D-glucan. Fig. 2 (right). Conformational energy surface for dimeric segment of homopolymeric $(1,3)$-linked $\beta$-D-glucan. Linkage torsion angles $\phi$ and $\psi$ are defined completely elsewhere $(10)$. Contour lines are drawn at $1,2,5,10$ and $50 \mathrm{kcal} / \mathrm{mol}$ relative to the lowest energy conformer marked with an $\mathrm{X}$.

The local trajectories of long glucan chains constructed from either $(1,2)$ - or $(1,3)$-linked dimeric segments (Figs. 3 and 4 ) are very different from one another, despite the similarity of the energy maps. The (1,3)- $\beta$-Dglucan displays a helical tendency; the (1,2)- $\beta$-D-glucan has been described as crumpled(11). The images in Figs. 3 and 4 were produced in a computer by adding glucose residues to a growing glucan chain and selecting the conformation of each successive glucose in $\phi, \psi$ space in accordance with the Boltzmann probablility distributions dictated, respectively, by Figs. 1 and 2. Only the glycosidic oxygens are shown. They are linked by virtual bond vectors that span the rigid sugar rings. This representation discloses clearly the inherent trajectory of the chain backbone. 
These are snapshots that freeze the chain in time. In reality its shape changes at a rate that would be readily perceptible, for a chain segment of the length shown, on the time scale of a few ns. A single chain adopts a large number of conformations over time; according to the ergodic hypothesis a large group of chains adopts the same distribution of conformations at any one instant. We call this collection of conformations the equilibrium spatial distribution of the polymer. Comparisons with experiment require averaging over a suitable "Monte Carlo" sample or ensemble of such conformations. Viewed from a distance the time averaged cloud of glycosidic oxygens will look rather similar for the two glucans, but the local trajectories will maintain a distinctly different character. Only information about the interactions of nearest neighboring sugars are included in making Figs. 3 and 4, so the volume from which one sugar ring excludes those to which it is not directly bonded is not taken into account. Some unrealistic chain conformations may therefore be generated. Longer range interactions can readily be taken into account at the expense of greater computation time, but doing so will not change the qualitative conclusions of the discussion to follow.
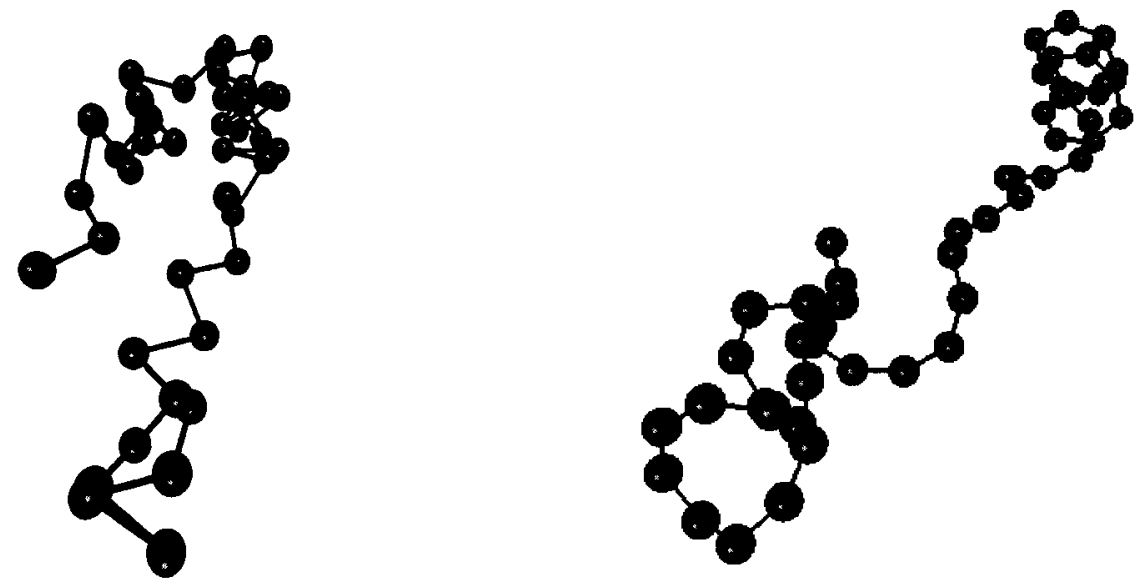

Fig. 3 (left). Local trajectory of long glucan chain constructed from $\beta-(1,2)$-linked dimeric segments. Fig. 4 (right). Local trajectory of long glucan chain constructed from $\beta-(1,3)$-linked dimeric segments. Sugar residues are spanned by virtual bonds, and only the glycosidic oxygens are shown.

Although $\beta$-glucans linked $(1,2)$ and $(1,3)$ are seen to have quite different chain trajectories, we might well expect the dynamics, i.e., the time dependence of the transformations among the various shapes characterizing the equilibrium distribution, to be rather similar, because the motions in $\phi, \psi$ space required to effect these shape changes must occur on essentially the same energy surface. Larger dynamic differences might be expected, however, between glucans linked $\alpha-(1,4)$ and $\alpha-(1,6)$. These linkages represent the extremes in glucan conformational freedom. The $\alpha-(1,4)$ is among the most conformationally constrained, and the $\alpha-(1,6)$, because of the extra chemical bond (C5-C6) in the linkage, has much greater inherent conformational freedom(2). The corresponding conformational energy surfaces are shown in Figs. 5 and 6 , and snapshots of the respective trajectories can be viewed in Figs. 2 and 3 of Brant et al( 4$)$. For the $\alpha-(1,6)$ linkage only the section in $\phi, \psi, \omega$ space at $\psi=180^{\circ}$ is shown in Fig. 6 , but this section is sufficient to demonstrate the conformational mobility associated with the C5-C6 torsion $\omega$ and the appreciable freedom in $\phi, \psi$ space throughout much of the range of $\omega$. The very large difference in the inherent conformational freedom of these two glucan linkages is perhaps best illustrated by comparing the decay of directional correlations between virtual bonds $i$ and $j$ as the sequential separation $j-i$ increases. This can be seen in Figs. 6 and 7 of Brant et al(4). Given the obvious difference in inherent conformational freedom, we consider below whether the rates of interconversion among conformations of the equilibrium spatial distribution of the $(1,4)-\alpha-D-$ and $(1,6)-\alpha-D$-glucan chains in solution might be different as well.

\section{POLYSACCHARIDE MODELS YIELD QUANTITATIVELY ACCURATE DESCRIPTIONS OF EQUILIBRIUM CHAIN DIMENSIONS}

It is appropriate at this point to examine the extent to which models of the sort employed here are capable of quantitative accuracy. Comparisons between the predictions of molecular modeling and the observables of X-ray crystallography and NMR spectroscopy are now commonplace(12). Crystallographic methods refer exclusively to structurally rigid molecules, so no information is available concerning single stranded 

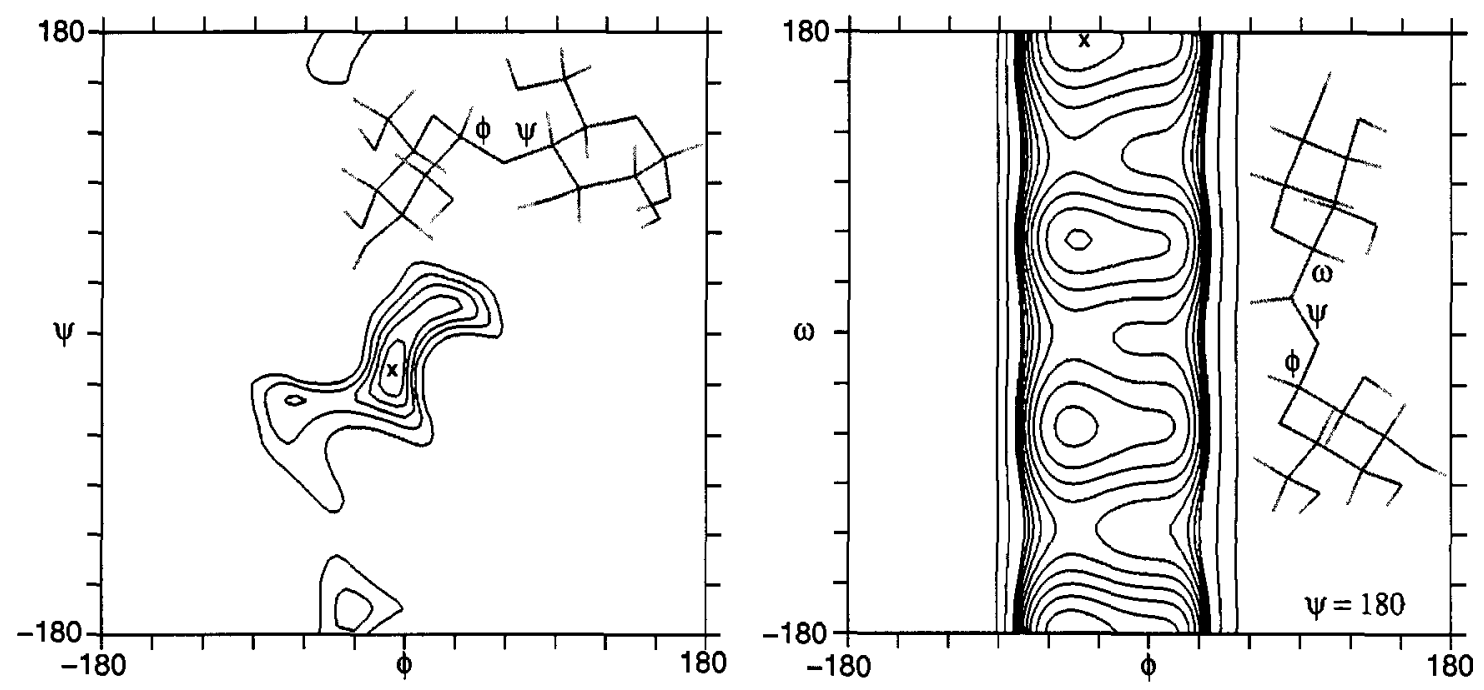

Fig. 5 (left). Conformational energy surface for dimeric segment of homopolymeric (1,4)-linked $\alpha$-D-glucan. Fig. 6 (right). Conformational energy surface for dimeric segment of homopolymeric $(1,6)$-linked $\alpha$-D-glucan. Torsion angles $\phi, \psi$ and $\omega$ are defined and contour lines are drawn as in Figs. 1 and 2.

polysaccharides in aqueous solution. NMR spectroscopic probes of intramolecular distances are not restricted to rigid molecules, but because their spatial range seldom exceeds $0.5 \mathrm{~nm}$, they are often insensitive to conformational freedom of the sort inherent in a sequence of glycosidic linkages. An appropriate and demanding test of these methods for the purposes at hand involves comparison of observed and predicted global measures of the dimensions or the dipolar or dielectric character of the polymer chain(13). We restrict attention here to measures of mean polymer chain dimensions that can be obtained with visible light scattering, scattering of shorter wavelength radiation, e.g., X-rays, neutrons, in the appropriate angular range, or hydrodynamic methods. These observables, dependent upon the effects of glycosidic linkage flexibility accumulated over large numbers of linkages, are highly sensitive to the detailed shape of the potential energy surface and not just to the location of the principal minimum or minima. The reliability of a particular model for global spatial observables can be severely tested by examining the extent to which it can reproduce the temperature dependence of the observables, because here the location, physical extent, and relative energy of the several minima come prominantly into play.
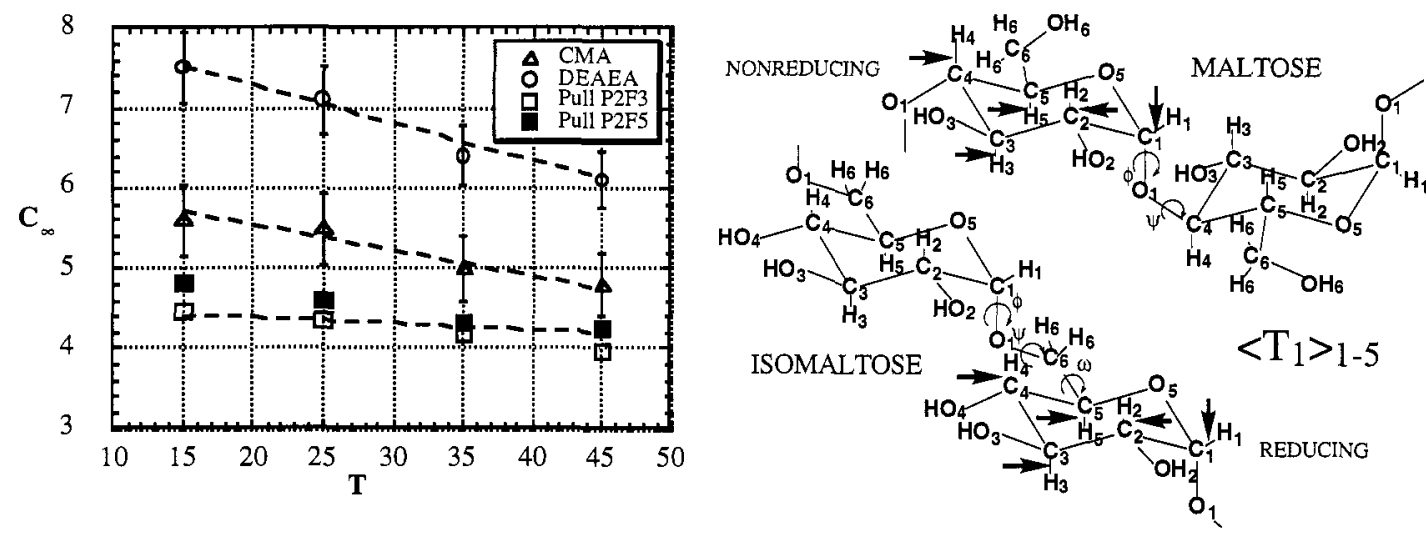

Fig. 7 (left). Measured values of the characteristic ratio $\mathrm{C}_{\infty}$ of carboxymethyl amylose (CMA), diethylaminoethyl amylose (DEAEA), and two fractions of pullulan (Pull). Experimental uncertainty for pullulan samples is comparable to that shown for the amylose derivatives. Theoretical fits are shown by dashed curves. Fig. 8 (right). Dimeric units "maltose" and "isomaltose" of the $\alpha-(1,4)$ - and $\alpha-(1,6)$-linked glucans amylose and dextran, respectively. The five $\mathrm{C}-\mathrm{H}$ vectors that contribute to the reported mean longitudinal relaxation time $\left\langle\mathrm{T}_{1}\right\rangle_{1-5}$ for each type of residue are shown as are the reducing and non-reducing ends of the dimers. 
Careful measurements of the temperature dependence of the chain dimensions of $\alpha$-D-glucans containing $(1,4)$ and $(1,6)$ linkages have been carried out in aqueous solution. Experimental results are shown in Fig. 7 for aqueous amylose, sparsely substituted with carboxymethyl and diethylaminoethyl groups to enhance solubility(14), and for the regularly repeating copolymer, pullulan(15). The characteristic ratio $\mathrm{C}_{\infty}$ is proportional to the mean square end-to-end distance of the polymer chain(13). Amylose is linked exclusively $(1,4)$, while in pullulan the linkages occur in the repeating sequence $(1,4)-(1,4)-(1,6)$. Statistical mechanical models, based on potential energy surfaces similar to those in Figs. 5 and 6, are able to reproduce these results essentially to within experimental error, as shown by the dashed lines in Fig. 7(16, 17). Because the experimental data refer to (theta) conditions under which the long range excluded volume of the polymer vanishes(18), neglect of long range interactions, implicit in the use of the disaccharide energy surfaces of Figs. 5 and 6, is permissible. Nevertheless, one cannot realistically expect that even the most carefully fashioned potential energy functions and parameter sets will be fully consistent with data of the sort shown in Fig. 7. Data sets used to train the potential functions come in general from disparate sources $(19,20,21,22,23)$, usually not including the theta solution behavior of polysaccharide chains, and seldom is any realistic account taken of the conformational perturbations due to the presence of solvent. Thus, the model-based curves in Fig. 7 are achieved with some modest manipulation, i.e., "refinement", of the potential functions to achieve the best possible fits to the data. It is therefore appropriate to regard the refined energy surfaces as potentials of mean force that are consistent with existing experimental results and that may reflect the essential physics of the system. The relative magnitudes of the individual terms contributing to the surface must, however, be interpreted with caution. Such surfaces may be used judiciously to predict chain properties not yet measured or not subject to measurement. In the same spirit, emergence of additional experimental data may dictate further revision of the model.

\section{CONFORMATIONAL MOTIONS CAN BE PROBED WITH NMR RELAXATION}

Given the near quantitative success of modeling the equilibrium chain dimensions of $\alpha$-D-glucans containing $(1,4)$ and $(1,6)$ linkages, we turn next to the question of the dynamics of conformational interchange among the countless chain conformations that characterize the equilibrium spatial distribution of glucans with these linkages. Attention is directed toward the (1,4)-linked and (1,6)-linked $\alpha$-D-glucans, amylose and dextran, respectively. We are interested chiefly in the collective motions of two, three, or more sugar residues bonded in sequence that occur principally through excursions along the coordinates $\phi, \psi$, (and $\omega)$ and, consequently, have the greatest influence on the local chain trajectory. These "segmental motions" of the polymer backbone occur on the ns timescale and can be probed using NMR relaxation in the 0.1 to $1 \mathrm{GHz}$ frequency range $(24,25,26)$.

Relaxation observables such as the longitudinal relaxation time $\left(\mathrm{T}_{1}\right)$, the transverse relaxation time $\left(\mathrm{T}_{2}\right)$, and the nuclear Overhauser enhancement (NOE) are most easily interpreted in terms of polysaccharide segmental motions when ${ }^{13} \mathrm{C}$ NMR is employed $(26,27)$. For ${ }^{13} \mathrm{C}$ NMR all of these effects depend to a good approximation only on the magnetic dipolar interaction of a ${ }^{13} \mathrm{C}$ nucleus and its directly bonded ${ }^{1} \mathrm{H}$ 's $(28)$. Relaxation of an excited ${ }^{13} \mathrm{C}$ nucleus is stimulated by fluctuations, at the proper frequencies, in the local magnetic field at the ${ }^{13} \mathrm{C}$ nucleus. These fluctuations arise from the random thermal motions of the directlybonded protons relative to the excited ${ }^{13} \mathrm{C}$. Specifically, it is the rate and form of the decay of the angular autocorrelation of the ${ }^{13} \mathrm{C}-{ }^{1} \mathrm{H}$ bond vectors in the laboratory coordinate system that determines the magnitudes of the relaxation observables(29).

The five $\mathrm{C}-\mathrm{H}$ vectors associated with the pyranose ring carbons of $(1,4)$ - and $(1,6)$-linked dimeric segments of amylose and dextran are highlighted with arrows in Fig. 8. It is evident that random motions along the coordinates $\phi, \psi$, (and $\omega$ ) will have an important influence on the persistence of the angular autocorrelation of these bond vectors, but, in fact, many kinds of motions contribute to the loss of correlation: vibrations of bonds about their mean lengths, vibrations of valence angles and librations of torsion angles about their mean values, conformational transformations involving a change in a torsion angle from one low energy domain on the potential surface to another, rotation of the macromolecule as a whole. The time scales of these motions range from fs for the most rapid bond vibrations to $\mathrm{ms}$ for the rotational relaxation of a large macromolcule as a whole. Most of the modes actually leading to loss of angular correlation are complicated, simultaneous combinations of these elementary motions. The faster modes contribute significantly to the decay in its intitial stages, but if the range of the motion is restricted, as, for example, with bond angle vibrations, they 
may be ineffective in producing complete decay of the correlation function on the necessary time scale. Very slow tumbling of the macromolecule as a whole can lead to complete loss of angular correlation, but such modes are much less efficient than those with characteristic times close to the inverse of the resonance frequency of the spectometer, as we now demonstrate.

Figure 9 shows the temporal decay of the angular autocorrelation function $\mathrm{G}(\tau, \mathrm{t})$ of a typical polysaccharide ${ }^{13} \mathrm{C}-{ }^{1} \mathrm{H}$ vector, assuming that the decay is exponential in time $\mathrm{t}$. Three different correlation times $(\tau=$ $0.03,0.3$, and $3.0 \mathrm{~ns}$ ) are illustrated. Because the motions responsible for the decay are complex, it is not required that the correlation be lost as a single exponential time decay. This simple time dependence has just been assumed here for purposes of illustration, because it is readily understood and subject to easy mathematical manipulation. In general, the correlation time is the ensemble averaged mean time required for the ${ }^{13} \mathrm{C}-{ }^{1} \mathrm{H}$ vector to have lost $63 \%$ of the memory of its original direction in space due to the thermal motions of the polymer chain.

Even a simple exponential decay is occasioned by a frequency spectrum of motions; a variety of essentially random events contribute to the process by which directional memory is lost. The frequency spectrum, or spectral density function, $J(\tau, v)$, of these random events is disclosed by Fourier transformation of the autocorrelation function, as shown in Fig. 10. Each of these spectral density functions is that of a simple exponential decay. The spectral density function associated with the decay of autocorrelation of a ${ }^{13} \mathrm{C}-{ }^{1} \mathrm{H}$ vector in a real molecule might be considerably more complicated in form, if a variety of processes operating on essentially different time scales contibute to the loss of directional memory. Notice, however, in Fig. 10 the immutable principle of relaxation: quickly decaying processes are characterized by broad spectral density functions, while slowly decaying processes are characterized by narrow spectral density functions. All of the spectral density functions in Fig. 10 are normalized to a common area under the curve.
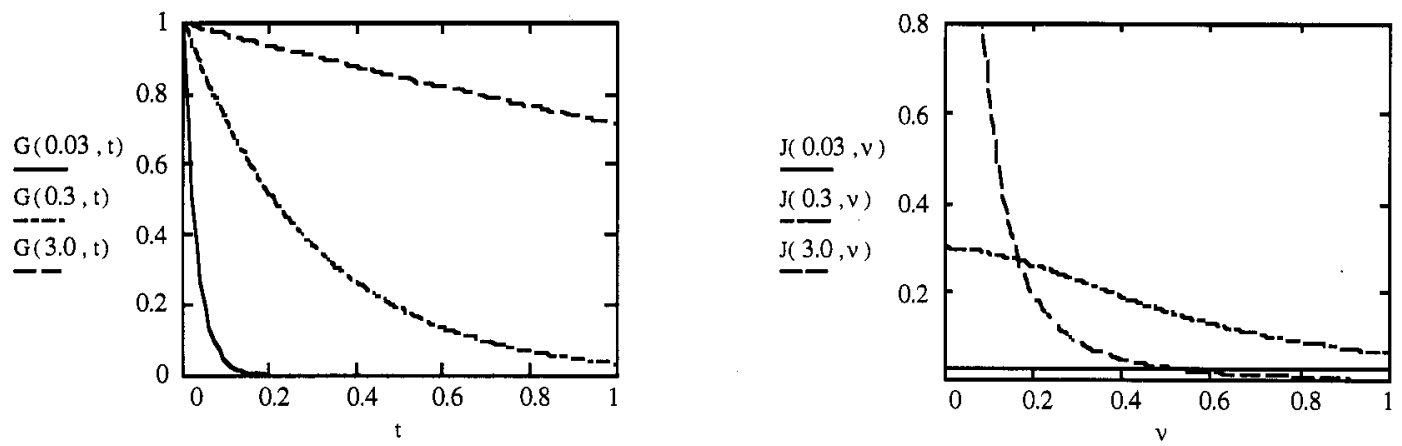

Fig. 9 (left). Time autocorrelation function $\mathrm{G}(\tau, \mathrm{t})$ for ${ }^{13} \mathrm{C}-{ }^{1} \mathrm{H}$ vector orientation plotted vs. time $\mathrm{t}$ in $\mathrm{ns}$ for three correlation times $(\tau=0.03,0.3$, and $3.0 \mathrm{~ns})$. Fig. 10 (right). Spectral density function $\mathrm{J}(\tau, \nu)$, obtained from the corresponding $G(\tau, t)$ by Fourier transformation, plotted against frequency $v$ in $\mathrm{GHz}$.

The key to ${ }^{13} \mathrm{C}$ NMR relaxation as a probe of polysaccharide motions lies in the relationships between the relaxation observables and $J(\tau, v)$. We confine attention here to $T_{1}$ and note that the inverse of $T_{1}$ is determined entirely by $J(\tau, v)$ at the specific frequencies $v_{H}, v_{H}+v_{C}$, and $v_{H}-v_{C}$, where $v_{C}$ and $v_{H}$ are the resonance frequencies of ${ }^{13} \mathrm{C}$ and ${ }^{1} \mathrm{H}$ at the field strength in question(29). The larger the spectral density at these frequencies, the more power available in the molecular motions for longitudinal relaxation of an excited ${ }^{13} \mathrm{C}$, and the shorter $\mathrm{T}_{1}$. For a spectrometer operating at $11.7 \mathrm{~T}$ this means that only the spectral densities at 375,500 , and $625 \mathrm{MHz}$ are significant for $\mathrm{T}_{1}$. Using $v_{\mathrm{H}}=500 \mathrm{MHz}=0.5 \mathrm{GHz}$ as an example, we see then from Fig. 10 that decay processes that are too slow ( $\tau>3 \mathrm{~ns}$ ) produce very narrow spectral density functions which have little power available at $0.5 \mathrm{GHz}$ to relax the excited ${ }^{13} \mathrm{C}$ nucleus. Likewise, decay processes that are too fast $(\tau<0.03 \mathrm{~ns})$ have broad and flat spectral density functions, again with little power available to relax the excited nucleus. Decay processes with characteristic times close to $\tau=0.3 \mathrm{~ns}=$ $0.15 / v_{\mathrm{H}}$ are those most effective in ${ }^{13} \mathrm{C}$ NMR longitudinal relaxation at this field strength. This is just the time scale of the segmental motions of the polymer backbone as anticipated at the outset. Other time scales could in principal be explored by changing the magnetic field and, hence, the resonance frequencies of the spectrometer. Unfortunately, spectral resolution diminishes as field strength is reduced, and the relaxation times of individual ${ }^{13} \mathrm{C}$ atoms become more difficult to measure. Access to higher frequency motions is limited by the availability and cost of higher field magnets. In practice the frequency range 0.1 to $1.0 \mathrm{GHz}$ is 
available. Other relaxation parameters may probe the spectral density function at other frequencies. In particular $\mathrm{T}_{2}$ also depends on the magnitude of $\mathrm{J}(\tau, v)$ at $v=0$. We will, however, restrict further attention here to measurements and interpretation of ${ }^{13} \mathrm{C} \mathrm{NMR} \mathrm{T}_{1}$ 's for oligomers related to amylose and dextran.

\section{MEASUREMENTS OF ${ }^{13}$ C NMR T 1 'S FOR OLIGOMERIC (1,4)- AND (1,6)-LINKED $\alpha$-D- GLUCANS}

The open symbols in Fig. 11 represent the measured ${ }^{13} \mathrm{C}$ NMR $\mathrm{T}_{1}$ 's associated with the glucose residues of the octameric $(1,4)$-linked $\alpha$-D-glucan plotted against residue number in the octamer(4). Data are reported for aqueous solutions at 22 and $65^{\circ} \mathrm{C}$ at a concentration $(3 \mathrm{~g} / \mathrm{dL})$, where $T_{1}$ is independent of concentration( $(30)$. The reported $T_{1}$ 's are the mean measured values for the five endocyclic carbons of each sugar ring; measured differences among the five ring carbons of any sugar were smaller than the experimental uncertainty $( \pm 5 \%)$. Terminal residues have longer $T_{1}$ 's than interior residues, and $T_{1}$ increases with increasing temperature. The measurements are not necessarily in the "extreme narrowing" limit but are clearly in the regime where increased mobility diminishes the rate of longitudinal relaxation. The reducing end of the oligomer has greater apparent mobility than the nonreducing end. Not shown here is the interesting observation that reducing terminal residues with the $\beta$-anomeric configuration have longer $T_{1}$ 's than those in the $\alpha$-anomeric form. The $\mathrm{T}_{1}$ 's of penultimate and interior residues were indistinguishable within experimental error. So, whereas terminal and penultimate residues are distinguishable on the basis of chemical shift from one another and from interior residues, only the terminal residues are distinguishable dynamically from the others. Hence, the plots of $T_{1}$ against residue number are horizontal from residue 2 through 7 . Interior residues of the octamer
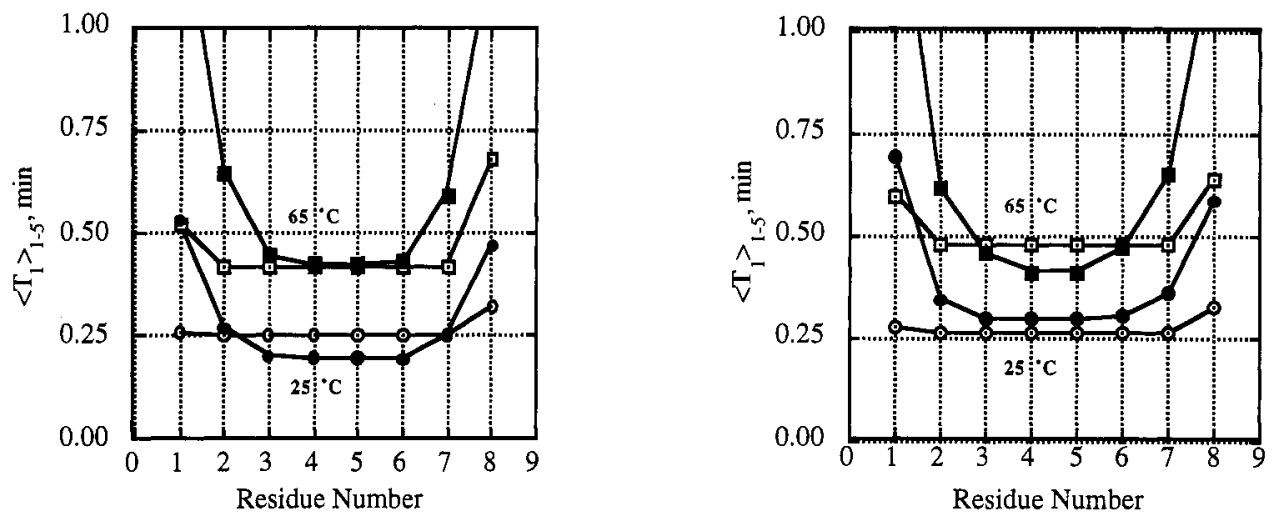

Fig. 11 (left). The mean $\mathrm{T}_{1}$ for the five endocyclic carbons of each residue of the octameric $\alpha-(1,4)$-glucan plotted against residue number at 22 and $65^{\circ} \mathrm{C}$ in aqueous solution at a concentration of $3 \mathrm{~g} / \mathrm{dL}$. Numbering of residues increases from the nonreducing toward the reducing end. Open symbols: experiment(4). Filled symbols: theory(31,32). Fig. 12 (right). Same as Fig. 11 but for the octameric $\alpha-(1,6)$-glucan.

behave dynamically, as probed by ${ }^{13} \mathrm{C} \mathrm{T}_{1}$ measurements, exactly as the interior residues of much longer oligomers and polymers (30). This suggests that the "wavelength" of the chain segment responsible for the segmental motions that produce ${ }^{13} \mathrm{C}$ NMR relaxation at $11.7 \mathrm{~T}$ in these glucans is of the order of $6-10$ residues. Exactly the same kind of data are presented in Fig. 12 for the octameric $(1,6)$-linked $\alpha$-D-glucan, and the same generalizations can be made.

Further experimental observations are summarized elsewhere(4). Most striking is the identity, within experimental uncertainty, of the $\mathrm{T}_{1}$ values at $22{ }^{\circ} \mathrm{C}$ for oligomers of the same DP from the malto- and isomaltooligomer series. Despite clear differences in the conformational energy surfaces governing segmental motions of the polymers, the dynamical behavior probed by ${ }^{13} \mathrm{C} \mathrm{NMR} \mathrm{T}_{1}$ 's is the same. This is tentatively interpreted as a dominance at $22^{\circ} \mathrm{C}$ of the viscous effects of the aqueous solvent, which are common to the two kinds of oligomers, over the structural effects implicit in the conformational energy surface. At temperatures sufficiently greater than $22^{\circ} \mathrm{C}$ one might expect that reduction in the solvent viscosity and the 
opportunity provided at the larger thermal energies for the molecules to explore a wider domain in the torsional configuration space would permit differences in the inherent conformational freedom of the glycosidic linkages to be revealed in the relative values of $T_{1}$ for the two series. These expectations are realized in the data at $65^{\circ} \mathrm{C}$. Specific features of the behavior a elevated temperatures, e.g., the apparent minimum in $\mathrm{T}_{1}$ at $\mathrm{DP}=6$ for the maltoligomer series, may resist facile interpretation. Likewise, an explanation of the dynamical differences between the reducing and nonreducing ends and the different dynamical behavior of $\alpha$ - and $\beta$-anomeric reducing termini will probably require modeling at a detailed level. An initial attempt to model the dynamical behavior of these chains using the optimized Rouse-Zimm local dynamics model $(31,32)$ shows clear success for interior residues of the oligomers and even reproduces rather nicely the temperature dependence of $T_{1}$; see the filled symbols in Figs. 11 and 12 .

\section{REFERENCES}

1. D. A. Brant, in The Biochemistry of Plants J. Preiss, Eds. (Academic Press, New York, 1980), vol. 3, pp. 425-472.

2. B. A. Burton, D. A. Brant, Biopolymers 22, 1769-1792 (1983).

3. D. A. Brant, M. D. Christ, in Computer Modeling of Carbohydrate Molecules A. D. French, J. W. Brady, Eds. (American Chemical Society, Washington, 1990), vol. 430, pp. 42-68.

4. D. A. Brant, H.-S. Liu, Z. S. Zhu, Carbohydrate Research 278, 11-26 (1995).

5. M. K. Dowd, P. J. Reilly, A. D. French, Biopolymers 34, 625-638 (1994).

6. M. Hricovini, G. Torri, Carbohydrate Research 268, 159-175 (1995).

7. A. D. French, V. Tran, Biopolymers 29, 1599-1611 (1990).

8. V. J. Morris, in Food Polysaccharides and Their Applications A. M. Stephen, Eds. (Marcel Dekker, New York, 1995) pp. 341-375.

9. D. A. Brant, Quarterly Reviews of Biophysics 9, 527-596 (1976).

10. M. Dentini, V. Crescenzi, D. Blasi, International Journal of Biological Macromolecules 6, 93-99 (1984).

11. D. A. Rees, W. E. Scott, Journal of the Chemical Society (B) , 469-479 (1971).

12. A. D. French, J. W. Brady, Eds., Computer Modeling of Carbohydrate Molecules, vol. 430 (American Chemical Society, Washington, 1990).

13. W. L. Mattice, U. W. Suter, Conformational Theory of Large Molecules (John Wiley \& Sons, New York, 1994).

14. K. D. Goebel, D. A. Brant, Macromolecules 3, 634-643 (1970).

15. G. S. Buliga, D. A. Brant, International Journal of Biological Macromolecules 9, 71-76 (1987).

16. D. A. Brant, W. L. Dimpfl, Macromolecules 3, 644-654 (1970).

17. G. S. Buliga, D. A. Brant, International Journal of Biological Macromolecules 9, 77-86 (1987).

18. P. J. Flory, Statistical Mechanics of Chain Molecules (Wiley-Interscience, New York, 1969).

19. S. N. Ha, A. Giammona, M. Field, J. W. Brady, Carbohydrate Research 180, $207-221$ (1988).

20. A. D. French, M. K. Dowd, Journal of Molecular Structure (Theochem) 105, 183-201 (1993).

21. T. M. Glennon, Y. J. Zheng, S. M. LeGrand, B. A. Shutzberg, K. M. Merz Jr., Journal of Computational Chemistry 15, 1019-1040 (1994).

22. M. L. C. E. Kouwijzer, P. D. J. Grootenhuis, Journal of Physical Chemistry 99, 13426-13436 (1995).

23. R. J. Woods, R. A. Dwek, C. J. Edge, B. Fraser-Reid, Journal of Physical Chemistry 99, 3832-3846 (1995).

24. F. Heatley, Progress in NMR Spectroscopy 13, 47-85 (1979).

25. F. A. Bovey, L. W. Jelinski, Journal of Physical Chemistry 89, 571-583 (1985).

26. A. J. Benesi, D. A. Brant, Macromolecules 18, 1109-1116 (1985).

27. P. Dais, Carbohydrate Research 160, 73-93 (1987).

28. J. W. Peng, G. Wagner, Journal of Magnetic Resonance 98, 308-332 (1992).

29. R. M. Levy, M. Karplus, P. G. Wolynes, Journal of the American Chemical Society 103, 5998-6011 (1981).

30. M. Kadkhodaei, H. Wu, D. A. Brant, Biopolymers 31, 1581-1592 (1991).

31. A. Perico, Accounts of Chemical Research 22, 336-342 (1989).

32. A. Perico, Biopolymers 28, 1527-1540 (1989).

Acknowledgements: The author thanks Jennifer H.-Y. Liu for assistance in preparing the figures for this paper and NIH Research Grant GM33062 for financial support of this research. 